\title{
ON THE CODING GAIN OF SEPARABLE 2D WAVELET FILTER BANKS
}

\author{
Michael D. Adams \\ Dept. of Elec. and Comp. Engineering, University of Victoria, Victoria, BC, V8W 3P6, Canada
}

\begin{abstract}
It has been observed that filter banks from a certain popular separable $2 \mathrm{D}$ wavelet class always appear to have a higher coding gain with respect to a separable image model than with respect to an isotropic one. This behavior is examined in detail and an explanation for it is offered. As it turns out, for such filter banks, it is highly improbable (if not impossible) for the isotropic coding gain to exceed the separable coding gain.
\end{abstract}

Index Terms - image coding, wavelet transforms, filter banks, coding gain, separable/isotropic image models

\section{INTRODUCTION}

Wavelet filter banks are frequently used for image coding (e.g., [1]). In this context, one important characteristic of a filter bank is its coding gain [2], which is often used as a criterion in optimal filterbank design. In earlier work, Xu and Adams [3] observed that, for a certain popular class of filter banks, the separable coding gain always seems to exceed the isotropic coding gain. In particular, this class is comprised of separable 2D wavelet filter banks constructed from 1D two-channel perfect-reconstruction (PR) real-coefficient filter banks, with at least one dual vanishing moment. In this paper, we study the coding gain of such filter banks in more detail and provide an explanation as to why the separable coding gain seems to always exceed the isotropic coding gain.

The remainder of the paper is structured as follows. Section 2 introduces some notational conventions used herein and Section 3 provides some background information on coding gain. Section 4 studies the coding gain in detail and presents a number of interesting results. Finally, Section 5 concludes the paper with a summary of our work.

\section{NOTATION AND TERMINOLOGY}

Before proceeding further, we introduce some of the notation employed herein. We denote the sets of integers, positive integers, and real numbers as $\mathbb{Z}, \mathbb{Z}^{+}$, and $\mathbb{R}$, respectively. The element of the $2 \mathrm{D}$ sequence $f$ with index $n=\left(n_{0}, n_{1}\right) \in \mathbb{Z}^{2}$ is denoted as either $f\left[n_{0}, n_{1}\right]$ or $f[n]$, whichever is more convenient. A similar notational convention is also employed for 2D functions. The Fourier transform of a sequence/function $f$ is denoted as $\hat{f}$. The symbols $*, \star$, and $\|\cdot\|$ denote convolution, correlation, and norm, respectively. The $l^{p}$ norm of $n=\left(n_{0}, n_{1}\right)$ is defined as $\left\|\left(n_{0}, n_{1}\right)\right\|_{l^{p}}=\left(\left|n_{0}\right|^{p}+\left|n_{1}\right|^{p}\right)^{1 / p}$, where $p \in\{1,2\}$ herein. Lastly, the sans-serif letters " $h$ " and " $v$ " are used to denote the horizontal and vertical components of separable functions/sequences/operators. For example, the horizontal and vertical components of the the separable 2D sequence $f$ would be denoted as $f_{\mathrm{h}}$ and $f_{\mathrm{v}}$, respectively (i.e., $f\left[n_{0}, n_{1}\right]=f_{\mathrm{h}}\left[n_{0}\right] f_{\mathrm{v}}\left[n_{1}\right]$ ).

This work was supported by the Natural Sciences and Engineering Research Council of Canada.

\section{CODING GAIN}

An $L$-level wavelet filter bank can always be equivalently represented as a nonuniform $m$-channel filter bank, where $m=3 L+1$. This equivalent $m$-channel filter bank consists of the analysis filters $\left\{h_{k}\right\}_{k=0}^{m-1}$, synthesis filters $\left\{g_{k}\right\}_{k=0}^{m-1}$, and downsamplers/upsamplers with sampling matrices $\left\{M_{k}\right\}_{k=0}^{m-1}$. Due to the separable nature of the filters, the subbands have four possible orientations: horizontallyand-vertically lowpass (LL), horizontally highpass and vertically lowpass (LH), horizontally lowpass and vertically highpass $(\mathrm{HL})$, and horizontally-and-vertically highpass $(\mathrm{HH})$. The coding gain $G$ of the above filter bank is given by [2]

$$
G=\prod_{k=0}^{m-1}\left(\frac{\alpha_{k}}{A_{k} B_{k}}\right)^{\alpha_{k}},
$$

where $A_{k}=\sum_{l \in \mathbb{Z}^{2}} \sum_{p \in \mathbb{Z}^{2}} h_{k}[l] h_{k}[p] r[p-l], B_{k}=\alpha_{k} \sum_{l \in \mathbb{Z}^{2}} g_{k}^{2}[l], \alpha_{k}=$ $\left|\operatorname{det} M_{k}\right|^{-1}$, and $r$ is the normalized autocorrelation sequence of the source image model. The two most common choices for $r$ are given by the separable and isotropic models, which are, respectively,

$$
r_{\text {sep }}[n]=\rho^{\|n\|_{l^{1}}} \quad \text { and } \quad r_{\text {iso }}[n]=\rho^{\|n\|_{l^{2}}},
$$

where $\rho$ is a correlation coefficient ${ }^{1}$ satisfying $0 \leq \rho \leq 1$. Since numerous quantities used herein depend on the image model (e.g., $G,\left\{A_{k}\right\}_{k=0}^{m-1}$ ), we use the qualifiers "sep" and "iso" to denote these quantities in the separable and isotropic cases, respectively. For example, $A_{k, \text { sep }}$ denotes the quantity $A_{k}$ given by (1) with $r=r_{\text {sep }}$ and $G_{\text {iso }}$ denotes the coding gain $G$ given by (1) with $r=r_{\text {iso }}$.

As an aside, we note that, for the class of filter banks being considered herein, if the constraint of having at least one dual vanishing moment is dropped, it is possible for $G_{\text {iso }}=G_{\text {sep }}$. For example, this result is trivially obtained by the PR system with the $1 \mathrm{D}$ prototype analysis filters $H_{0}(z)=1$ and $H_{1}(z)=z$. So, obviously, for filter banks outside the class being considered herein, the condition $G_{\text {sep }}>G_{\text {iso }}$ may be violated.

\section{ANALYSIS}

We begin by making a few observations regarding the quantities $\left\{A_{k}\right\}_{k=0}^{m-1}$ and $\left\{B_{k}\right\}_{k=0}^{m-1}$ which appear in (1). Since each of the $\left\{A_{k}\right\}_{k=0}^{m-1}$ is the ratio between two variances (which are nonnegative real numbers), $A_{k} \geq 0$. For similar reasons, each of the $\left\{B_{k}\right\}_{k=0}^{m-1}$ satisfies $B_{k} \geq 0$. Furthermore, $A_{k}=0$ implies that $h_{k}$ is the zero sequence, and $B_{k}=0$ implies that $g_{k}$ is the zero sequence. Since, in practice, we never choose any of the $\left\{h_{k}\right\}_{k=0}^{m-1}$ or $\left\{g_{k}\right\}_{k=0}^{m-1}$ to be a zero sequence, we can safely assume that $A_{k}>0$ and $B_{k}>0$.

Next, we make a comment concerning the correlation coefficient $\rho$ appearing in (2). In the remainder of this paper, we exclude the

\footnotetext{
${ }^{1}$ To be more precise, in the separable case, it is also permissible to have $\rho \in[-1,0)$. Since $\rho$ cannot be negative in the isotropic case, we only consider nonnegative $\rho$ herein.
} 
possibility that $\rho \in\{0,1\}$, since these cases are of no practical value and only serve to complicate the subsequent analysis. Since image data is typically best modelled by $\rho$ satisfying $0.90 \leq \rho \leq 0.95$, we emphasize this range of values in most of our analysis.

From (1), we observe that the only dependence that the coding gain $G$ has on the image model embodied by $r$ is in the value of the $\left\{A_{k}\right\}_{k=0}^{m-1}$. Since $\alpha_{k}>0, G$ increases as one or more of the $\left\{A_{k}\right\}_{k=0}^{m-1}$ decrease. With this in mind, we would like to more carefully consider how the $\left\{A_{k}\right\}_{k=0}^{m-1}$ are affected by the choice of image model.

Before proceeding further, we need to define several new quantities that will be used throughout the remainder of this paper. The $k$ th factor in the product $G$ from (1) is denoted as $G_{k}$ (i.e., $G_{k}=$ $\left.\left(\frac{\alpha_{k}}{A_{k} B_{k}}\right)^{\alpha_{k}}\right)$. Hence, $G=\prod_{k=0}^{m-1} G_{k}$. Let $G_{k, \text { sep }}$ and $G_{k \text {,iso denote the }}$ quantity $G_{k}$ in the separable and isotropic cases, respectively. Define

$$
\begin{gathered}
\Delta A_{k}=A_{k, \text { iso }}-A_{k, \text { sep }}, \quad \Delta r=r_{\text {iso }}-r_{\text {sep }}, \quad \text { and } \\
\tilde{G}_{k}=G_{k, \text { iso }} / G_{k, \text { sep }} .
\end{gathered}
$$

\subsection{Time-Domain Analysis}

To begin, we examine how the choice of image model affects $r$ by considering the relationship between $r_{\text {sep }}$ and $r_{\text {iso }}$. To begin, we recall the following relationship between the $l^{1}$ and $l^{2}$ norms:

$$
\left\|\left(n_{0}, n_{1}\right)\right\|_{l^{1}} \geq\left\|\left(n_{0}, n_{1}\right)\right\|_{l^{2}} \quad \text { for all }\left(n_{0}, n_{1}\right) \in \mathbb{R}^{2}
$$

with equality holding if and only if $n_{0} n_{1}=0$ (i.e., at least one of $\left\{n_{0}, n_{1}\right\}$ is zero). Now, let $n=\left(n_{0}, n_{1}\right)$ and consider $r[n]$ for the separable and isotropic cases (i.e., $r=r_{\text {sep }}$ and $r=r_{\text {iso }}$, respectively). If $n_{0} n_{1}=0$, then we trivially have from (3) that $r_{\text {sep }}[n]=r_{\text {iso }}[n]$. Suppose now that $n_{0} n_{1} \neq 0$. Then from (3), $\|n\|_{l^{1}}>\|n\|_{l^{2}}$, and since $0<\rho<1$, this implies that $\rho^{\|n\|_{l^{1}}}<\rho^{\|n\|_{l^{2}}}$. Thus, $r_{\text {sep }}$ and $r_{\text {iso }}$ are related as follows:

$$
\text { for all }\left(n_{0}, n_{1}\right) \in \mathbb{Z}^{2}, \begin{cases}r_{\mathrm{sep}}\left[n_{0}, n_{1}\right]=r_{\text {iso }}\left[n_{0}, n_{1}\right] & \text { if } n_{0} n_{1}=0 \\ r_{\mathrm{sep}}\left[n_{0}, n_{1}\right]<r_{\text {iso }}\left[n_{0}, n_{1}\right] & \text { otherwise, }\end{cases}
$$

which implies $r_{\text {sep }}[n] \leq r_{\text {iso }}[n]$ for all $n \in \mathbb{Z}^{2}$. One can easily verify that the sequence $r$ has the following 8-fold symmetry in both the separable and isotropic cases (i.e., $r=r_{\text {sep }}$ and $r=r_{\text {iso }}$, respectively):

$$
\begin{gathered}
r\left[n_{0}, n_{1}\right]=r\left[-n_{0}, n_{1}\right]=r\left[n_{0},-n_{1}\right]=r\left[-n_{0},-n_{1}\right] \quad \text { and } \\
r\left[n_{0}, n_{1}\right]=r\left[n_{1}, n_{0}\right] .
\end{gathered}
$$

For later reference, the sequence $\Delta r$ (i.e., $r_{\text {iso }}-r_{\text {sep }}$ ) is plotted for $\rho=0.95$ in Fig. 2. For every $\rho \in[0.90,0.95]$, the basic shape of the function $\Delta r$ does not change, although as $\rho$ increases, the decay rate of $\Delta r$ decreases.

Consider the expression for $\left\{A_{k}\right\}_{k=0}^{m-1}$ given by (1). Through a change of variable, we can rewrite this expression in a more convenient form as

$$
A_{k}=\sum_{p \in \mathbb{Z}^{2}} r[p] c_{k}[p],
$$

where $c_{k}=h_{k} \star h_{k}$ (i.e., $c_{k}$ is the autocorrelation of $h_{k}$ ).

Since all of the filters $\left\{h_{k}\right\}_{k=0}^{m-1}$ are separable, their autocorrelation sequences $\left\{c_{k}\right\}_{k=0}^{m-1}$ are also separable. Due to the separability of $h_{k}$, the sequence $c_{k}$ has quadrantal symmetry:

$$
c_{k}\left[n_{0}, n_{1}\right]=c_{k}\left[-n_{0}, n_{1}\right]=c_{k}\left[n_{0},-n_{1}\right]=c_{k}\left[-n_{0},-n_{1}\right] .
$$

Using the quadrantal symmetry of $r$ and $c_{k}$, we can rewrite (6) as

$$
\begin{aligned}
A_{k}= & r[0] c_{k}[0]+2 \sum_{d_{0} \in \mathbb{Z}^{+}} r\left[d_{0}, 0\right] c_{k}\left[d_{0}, 0\right]+2 \sum_{d_{1} \in \mathbb{Z}^{+}} r\left[0, d_{1}\right] c_{k}\left[0, d_{1}\right] \\
& +4 \sum_{d \in\left(\mathbb{Z}^{+}\right)^{2}} r[d] c_{k}[d] .
\end{aligned}
$$

Due to the equality case of (4), only the last summation in the preceding expression for $A_{k}$ differs in the separable and isotropic cases. Thus, we have that

$$
\Delta A_{k}=4 \sum_{d \in\left(\mathbb{Z}^{+}\right)^{2}} \Delta r[d] c_{k}[d] .
$$

Let us now examine the preceding expression for $\Delta A_{k}$ (in (7)) in more detail. First, we make some observations about $\Delta r[d]$ for $d \in\left(\mathbb{Z}^{+}\right)^{2}$. From the inequality case in (4), we have that $\Delta r[d]>0$. Also, as can be seen from the plot of $\Delta r$ in Fig. 2, $\Delta r$ decreases with distance from the origin and $\Delta r\left[d_{0}, d_{1}\right]$ is larger when $d_{0}=d_{1}$ (i.e., along a line with an orientation of $\frac{\pi}{4}$ ). Next, we make an observation regarding $c_{k}$. Assuming that the filter $h_{k}$ has a reasonable frequency response, the sequence $c_{k}$ should decrease with distance from the origin (i.e., $c_{k, \mathrm{~h}}[n]$ and $c_{k, \mathrm{v}}[n]$ should have a decay of approximately $O\left(|n|^{-1}\right)$ ). Based on the preceding observations, one would expect many of the largest-magnitude terms in the summation in (7) to be obtained when $d=\left(d_{0}, d_{1}\right)$ is close to the origin and $d_{0}=d_{1}$. Thus, the sign of $c_{k}[d]$ for such values of $d$ should have a relatively strong influence on the sign of $\Delta A_{k}$. Furthermore, if the horizontal and vertical filters used to compose $h_{k}$ are the same (i.e., $h_{k, \mathrm{~h}}=h_{k, \mathrm{v}}$ ) one can easily confirm that $c_{k}\left[n_{0}, n_{0}\right] \geq 0$ for all $n_{0} \in \mathbb{Z}$. Thus, in this case, there would be a better likelihood for $\Delta A_{k}$ to be positive. At this point, we note that the filters $h_{k}$ associated with $\mathrm{LL}$ and $\mathrm{HH}$ subband orientations have $h_{k, \mathrm{~h}}=h_{k, \mathrm{v}}$. Therefore, for the reason just mentioned, $\Delta A_{k}$ is more likely to be positive for subbands with these orientations, leading to a tendency to favor $G_{\text {sep }}$ over $G_{\text {iso. As we }}$ will see later, this tendency turns out to be particularly strong in the case of the $\mathrm{HH}$ subbands.

We can make one further observation regarding $\Delta A_{k}$ in (7). Since $\Delta r[d]>0$, if $h_{k}[n] \geq 0$ for all $n \in \mathbb{Z}^{2}$ (or $h_{k}[n] \leq 0$ ), then $c_{k} \geq 0$, implying that $\Delta A_{k}>0$. Thus, $G_{\text {sep }}$ is favored over $G_{\text {iso }}$. This trivial case, however, can only occur for the LL subband orientation (since this scenario implies that the filter $h_{k}$ has a nonzero DC gain). Furthermore, this situation only seems likely to occur in practice for short filters. For this reason, this observation is not as interesting as the one in the previous paragraph.

Although we could present additional details here based on a time-domain analysis, we instead shift our focus to the frequency domain. As it turns out, some very valuable insights can be gained from a frequency-domain analysis.

\subsection{Frequency-Domain Analysis}

In anticipation of what is to come, we compute $\hat{r}$ for the separable and isotropic cases. The Fourier transform of $r_{\text {sep }}$ can be shown to be

$$
\hat{r}_{\mathrm{sep}}\left(\omega_{0}, \omega_{1}\right)=\frac{\left(1-\rho^{2}\right)^{2}}{\left(1-2 \rho \cos \omega_{0}+\rho^{2}\right)\left(1-2 \rho \cos \omega_{1}+\rho^{2}\right)} .
$$

Now, we consider the Fourier transform of $r_{\text {iso. }}$. Using the 8-fold symmetry of $r_{\text {iso }}$ (i.e., (5)), we can show that

$$
\begin{gathered}
\hat{r}_{\text {iso }}\left(\omega_{0}, \omega_{1}\right)=1+\gamma\left(\omega_{0}, \rho\right)+\gamma\left(\omega_{1}, \rho\right)+\gamma\left(\omega_{0}+\omega_{1}, \rho^{\sqrt{2}}\right) \\
+\gamma\left(\omega_{1}-\omega_{0}, \rho^{\sqrt{2}}\right)+2 \sum_{i=2}^{\infty} \sum_{k=1}^{i-1} \rho^{\sqrt{i^{2}+k^{2}}}\left(\cos \left(i \omega_{0}+k \omega_{1}\right)\right. \\
\left.+\cos \left(k \omega_{0}+i \omega_{1}\right)+\cos \left(k \omega_{0}-i \omega_{1}\right)+\cos \left(i \omega_{0}-k \omega_{1}\right)\right),
\end{gathered}
$$

where $\gamma(\omega, \alpha)=\frac{2 \alpha \cos \omega-2 \alpha^{2}}{1-2 \alpha \cos \omega+\alpha^{2}}$. In both the separable and isotropic cases, one can confirm from (8) and (9) that $\hat{r}$ has the following 8fold symmetry:

$$
\begin{gathered}
\hat{r}\left(\omega_{0}, \omega_{1}\right)=\hat{r}\left(-\omega_{0}, \omega_{1}\right)=\hat{r}\left(\omega_{0},-\omega_{1}\right)=\hat{r}\left(-\omega_{0},-\omega_{1}\right) \quad \text { and } \\
\hat{r}\left(\omega_{0}, \omega_{1}\right)=\hat{r}\left(\omega_{1}, \omega_{0}\right) .
\end{gathered}
$$

As an aside, we comment on an alternative scheme for computing $\hat{r}_{\text {iso }}$. By observing that the Fourier transform of the function 
$f(t)=\rho^{\|t\|_{l^{2}}}$ is $\hat{f}(\omega)=-2 \pi\left(\ln ^{2} \rho+\|\omega\|_{l^{2}}^{2}\right)^{-3 / 2} \ln \rho$ [4, Eqn. (3)] and $r_{\text {iso }}$ is a sampled version of $f$, we can conclude $\hat{r}_{\text {iso }}(\omega)=$ $-2 \pi(\ln \rho) \sum_{k \in \mathbb{Z}^{2}}\left[\ln ^{2} \rho+\|\omega-2 \pi k\|_{l^{2}}^{2}\right]^{-3 / 2}$. For computational purposes, this formula for $\hat{r}_{\text {iso }}$ is probably less useful than (9), due to an apparent slower convergence rate.

For future reference, $\hat{r}_{\text {sep }}$ and $\hat{r}_{\text {iso }}$ are plotted in Figs. 3 and 4 for $\rho=0.95$. For each of $\hat{r}_{\text {sep }}$ and $\hat{r}_{\text {iso }}$, the general shape of the plot remains the same for all $\rho \in[0.90,0.95]$, but the decay rate increases with increasing $\rho$. Also, $\widehat{\Delta r}$ is plotted for $\rho=0.95$ in Fig. 5. The gray-shaded region in the contour plot corresponds to where the plotted function is negative. As $\rho$ increases, the decay rate of $\widehat{\Delta r}$ increases, and there is a larger region where $\widehat{\Delta r}$ is positive. In all of the above plots, the horizontal and vertical axes are scaled by $\frac{1}{\pi}$ so 1 corresponds to the Nyquist frequency.

Now, we seek to characterize $A_{k}$ and $\Delta A_{k}$ in the frequency domain. To accomplish this, we use (6) and the fact that Fourier transform preserves inner products to write

$$
A_{k}=\frac{1}{4 \pi^{2}} \int_{[-\pi, \pi)^{2}} \hat{r}(\omega) \hat{c}_{k}(\omega) d \omega=\frac{1}{4 \pi^{2}} \int_{[-\pi, \pi)^{2}} \hat{r}(\omega)\left|\hat{h}_{k}(\omega)\right|^{2} d \omega .
$$

In the above simplification, we have that $\hat{c}_{k}(\omega)=\left|\hat{h}_{k}(\omega)\right|^{2}$ since $h_{k}$ is real. Due to the quadrantal symmetry of $\hat{r}$ and $\left|\hat{h}_{k}\right|^{2}$, we can rewrite the preceding equation as

$$
A_{k}=\frac{1}{\pi^{2}} \int_{[0, \pi)^{2}} \hat{r}(\omega)\left|\hat{h}_{k}(\omega)\right|^{2} d \omega
$$

Thus, we have that

$$
\Delta A_{k}=\frac{1}{\pi^{2}} \int_{[0, \pi)^{2}} \widehat{\Delta r}(\omega)\left|\hat{h}_{k}(\omega)\right|^{2} d \omega .
$$

Now, we will use (10) and (11) in order to gain some additional insight into the coding gain. In what follows, $L$ and $m$ are as defined in Section 3 (with $m=3 L+1$ ), and $k$ denotes the channel index where $k \in\{0,1,2, \ldots, 3 L\}$. The level in the analysis filter-bank tree associated with channel $k$ is denoted as level $(k)$. For the $l$ th level in the filter-bank tree, $\mathrm{LL}_{l}, \mathrm{LH}_{l}, \mathrm{HL}_{l}$, and $\mathrm{HH}_{l}$ denote the subbands with LL, LH, HL, and HH orientations, respectively. For convenience, we number the channels such that larger values of $k$ correspond to a deeper descent into the analysis filter-bank tree. Note that this numbering convention is backwards from what is typically used. That is, the lowest frequency (LL) band is always associated with the largest channel index $3 L$ (instead of the smallest one 0 ). With our convention, decompositions with $L$ and $L^{\prime}$ levels have an identical numbering scheme for their common filters/subbands. This allows for a much more concise presentation of some of our later results. The correspondence between channels and frequency bands is illustrated in Fig. 1, with the analysis filter associated with each subband being shown in parentheses. In order to avoid an overly complicated diagram, only the first quadrant of the frequency plane is shown, with the remainder following from (quadrantal) symmetry.

For two filter banks, we have computed the various coding-gainrelated quantities for $L \in\{1,2,3\}$ with $\rho=0.95$, the results of which can be found in Table 1. The first filter bank is an orthonormal system with ideal filters. This corresponds to a system with $1 \mathrm{D}$ prototype filters each having a gain of $\sqrt{2}$ and 0 in their passbands and stopbands, respectively. (Thus, we have that $h_{k}$ has passband and stopband gains of $2^{\text {level }(k)+1}$ and 0 , respectively.) The other filter bank is the well-known Haar system, and was chosen for comparison purposes as an example of a system with nonideal filters. The nonideal filter bank is normalized such that its $1 \mathrm{D}$ prototype filters have DC/Nyquist gains of $\sqrt{2}$, as this facilitates more direct comparisons with the ideal filter bank under consideration. Note that there

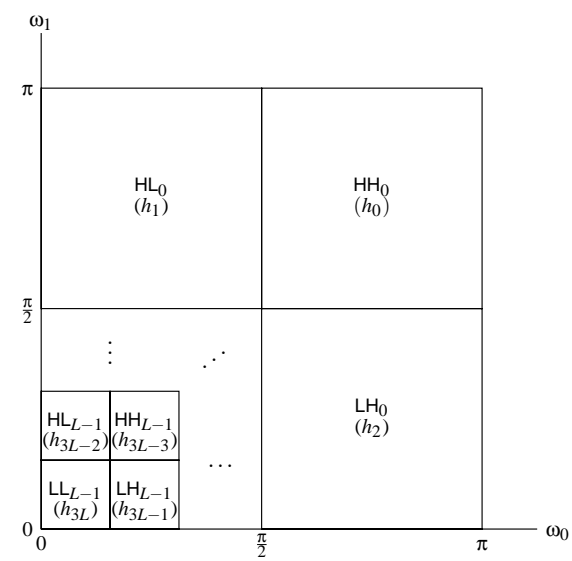

Fig. 1. Subband tiling of the first quadrant of the frequency plane.

is no loss of generality in considering only this particular normalization, as the coding gain for PR filter banks is invariant to scaling and translation of the analysis/synthesis filter impulse responses. We will refer to the results in the above tables in some of the discussion that follows.

Now, we more carefully examine the expression for the coding gain in (1). As $k$ increases (which corresponds to level $(k)$ increasing), $\alpha_{k}$ decays exponentially to zero (i.e., $\alpha_{k}=4^{-\operatorname{level}(k)-1}$ ). This implies that $G_{k} \rightarrow 1$ as $k$ increases. Thus (since $G=\prod_{k=0}^{m-1} G_{k}$ ), the most significant contributions to the coding gain $G$ come from $G_{k}$ associated with small $k$, especially those $k$ associated with the 0 th level (i.e., $k \in\{0,1,2\}$ or $k \in\{0,1,2,3\}$ for $L \geq 2$ and $L=1$, respectively). As $k$ increases, $\tilde{G}_{k} \rightarrow 1$. Thus (since $G_{\text {iso }} / G_{\text {sep }}=\prod_{k=0}^{m-1} \tilde{G}_{k}$ ), any difference in the separable and isotropic coding gains is most strongly influenced by $\tilde{G}_{k}$ for small $k$. Furthermore, simple algebraic manipulation shows that

$$
\tilde{G}_{k}=\left(\frac{A_{k, \mathrm{sep}}}{A_{k, \mathrm{sep}}+\Delta A_{k}}\right)^{\alpha_{k}}=\left(1 /\left(1+\frac{\Delta A_{k}}{A_{k, \mathrm{sep}}}\right)\right)^{\alpha_{k}} .
$$

Consequently, $G_{k, \text { sep }}$ and $G_{k \text {,iso }}$ differ most when $\left|\Delta A_{k}\right|$ is large relative to $A_{k, \text { sep }}$ (i.e., $\frac{\left|\Delta A_{k}\right|}{A_{k, \text { sep }}}$ is large). All of the above observations can be seen to be consistent with the data in Table 1 .

In what follows, it is instructive to consider the ideal filter bank introduced above. In this case, from (10) and (11), $A_{k}$ and $\Delta A_{k}$ are simply (up to scale) the integral of $\hat{r}$ and $\widehat{\Delta r}$ over the first-quadrant portion of the passband of the ideal filter $h_{k}$. To envision what the result of such integrations will be, it is helpful to imagine the frequency-plane tiling of Fig. 1 superimposed on the first quadrants of the plots of $\hat{r}_{\text {sep }}, \hat{r}_{\text {iso }}$, and $\widehat{\Delta r}$ in Figs. 3 , 4, and 5.

Consider $k=0$ (i.e., the $\mathrm{HH}_{0}$ band) in the case of the ideal filter bank. For $\omega$ in the $\left(\mathrm{HH}_{0}\right)$ passband, $\hat{r}_{\text {sep }}(\omega)$ and $\hat{r}_{\text {iso }}(\omega)$ are both very small, so $A_{k}$ is small, but $|\widehat{\Delta r}(\omega)|$ is large compared to $\hat{r}$ so $\Delta A_{k} / A_{k, \text { sep }}$ is large. Consequently, from (12), $\tilde{G}_{k}$ is small, meaning that $G_{\text {sep }}$ is very strongly favored over $G_{\text {iso }}$. A similar argument also applies to the other $\mathrm{HH}$ bands, but the influence on the coding gain $G$ is less significant in these cases since $\tilde{G}_{k} \rightarrow 1$ as $k$ increases. Now consider what happens in the case of nonideal filters. Even in this case, it is very difficult for the above qualitative behavior to change. To obtain significantly different behavior, $\hat{h}_{k}$ would have to be very large along the axes where $\widehat{\Delta r}$ is most negative (and therefore most favorable to higher $G_{\text {iso }}$ ). Due to the presence of at least one dual vanishing moment, however, $\hat{h}_{k}(\omega)$ must be zero along both axes. So, even in case of nonideal filters, $G_{\text {sep }}$ is still likely to be strongly 
favored by the $\mathrm{HH}$ bands.

In the case of the $\mathrm{LH}$ and $\mathrm{HL}$ bands, due to the presence of at least one dual vanishing moment, $\hat{h}_{k}\left(\omega_{0}, \omega_{1}\right)$ must be zero along exactly one of $\omega_{0}=0$ or $\omega_{1}=0$. We observe that $\widehat{\Delta r}$ (shown in Fig. 5) is largest in magnitude along the axes (with large positive values near the origin and large negative values elsewhere on the axes). Thus, in the calculation of $\Delta A_{k}$, the large positive values near the origin in $\widehat{\Delta r}$ are effectively cancelled, and we are left with large negative values along one axis. This leads to $\tilde{G}_{k}>1$ and the $\mathrm{LH}$ and $\mathrm{HL}$ bands favoring $G_{\text {iso }}$ over $G_{\text {sep }}$. Lastly, we note that the $\mathrm{LH}$ and $\mathrm{HL}$ subbands do not have as much impact on the coding gain $G$ as the $\mathrm{HH}$ bands. This is due to that fact $A_{k \text {,sep }}$ is larger for the $\mathrm{LH}$ and $\mathrm{HL}$ subbands, making it more difficult for $\left|\Delta A_{k}\right|$ to be large relative to $A_{k, \text { sep }}$.

An examination of the data for the nonideal filter bank in Table 1(b) shows that all of observations made above are consistent with this data. For example, the coding gain is most strongly influenced by the $\mathrm{HH}$ bands, with $\mathrm{HH}_{0}$ figuring most prominently. The $\mathrm{LL}$ and $\mathrm{HH}$ bands favor $G_{\text {sep }}$ (i.e., $\tilde{G}_{k}<1$ ), while the $\mathrm{LH}$ and $\mathrm{HL}$ bands favor $G_{\text {iso }}$ (i.e., $\tilde{G}_{k}>1$ ). The fact that $\mathrm{LL}$ and $\mathrm{HH}$ subbands favor $G_{\text {sep }}$ over $G_{\text {iso }}$ is also consistent with the results of our time-domain analysis earlier in Section 4.1.

\subsection{Additional Commentary}

In addition to the filter banks for which results are presented herein, we have also considered quite a number of other filter banks, some of which were considered for adoption in the JPEG-2000 standard (including the $9 / 7$ system [1]), while others were produced at various stages of the optimal design method of [3]. Similar trends (to those described above) were also found in the case of these other filter banks. Moreover, we were not able to find any filter bank (belonging to the class of filter banks under consideration in this paper) for which $G_{\text {iso }}>G_{\text {sep }}$. Based on our analysis herein, there is good reason to believe that such filter banks probably do not exist.

\section{CONCLUSIONS}

In this paper, we have studied the coding gain of separable 2D wavelet filter banks derived from 1D two-channel real-coefficient PR filter banks with at least one dual vanishing moment. We have explained why, for such filter banks, it is extremely difficult (if not impossible) for the isotropic coding gain to exceed the separable coding gain. The new insight provided by our analysis may prove helpful in the design of improved filter banks for image coding. For example, if one is trying to simultaneously maximize the minimum of $G_{\text {sep }}$ and $G_{\text {iso }}$ as in [3], our analysis shows that this is practically equivalent to optimizing $G_{\text {iso }}$ alone.

\section{REFERENCES}

[1] ISO/IEC 15444-1: Information technology-JPEG 2000 image coding system-Part 1: Core coding system, 2000.

[2] J. Katto and Y. Yasuda, "Performance evaluation of subband coding and optimization of its filter coefficients," in SPIE VCIP, 1991, vol. 1605, pp. 95-106.

[3] D. Xu and M. D. Adams, "Design of high-performance filter banks for image coding," in Proc. of IEEE ISSPIT, Vancouver, BC, Canada, Aug. 2006, pp. 868-873.

[4] J. B. O'Neal and T. R. Natarajan, "Coding isotropic images," IEEE Trans. on Information Theory, vol. 23, no. 6, pp. 697-707, Nov. 1977.
Table 1. Coding gain quantities for the (a) ideal and (b) Haar filter banks with $\rho=0.95$.

(a)

\begin{tabular}{|l|l|r|r|r|r|r|r|r|r|}
\hline$k$ & Band & $A_{k, \text { sep }}$ & $A_{k, \text { iso }}$ & $\Delta A_{k}$ & $\frac{\Delta A_{k}}{A_{k, s e p}}$ & $\frac{1}{\alpha_{k}}$ & $G_{k, \text { sep }}$ & $G_{k, \text { iso }}$ & $\tilde{G}_{k}$ \\
\hline 0 & $\mathrm{HH}_{0}$ & 0.001 & 0.025 & 0.024 & 22.85 & 4 & 5.53 & 2.50 & 0.45 \\
1 & $\mathrm{HL}_{0}$ & 0.064 & 0.039 & -0.024 & -0.378 & 4 & 1.98 & 2.23 & 1.12 \\
2 & $\mathrm{LH}_{0}$ & 0.064 & 0.039 & -0.024 & -0.378 & 4 & 1.98 & 2.23 & 1.12 \\
3 & $\mathrm{LL}_{0}$ & 3.870 & 3.894 & 0.024 & 0.006 & 4 & 0.71 & 0.71 & 0.99 \\
\hline 3 & $\mathrm{HH}_{1}$ & 0.004 & 0.050 & 0.046 & 10.95 & 16 & 1.40 & 1.20 & 0.85 \\
4 & $\mathrm{HL}_{1}$ & 0.250 & 0.132 & -0.117 & -0.470 & 16 & 1.09 & 1.13 & 1.04 \\
5 & $\mathrm{LH}_{1}$ & 0.250 & 0.132 & -0.117 & -0.470 & 16 & 1.09 & 1.13 & 1.04 \\
6 & $\mathrm{LL}_{1}$ & 14.76 & 15.07 & 0.307 & 0.020 & 16 & 0.84 & 0.84 & 0.99 \\
\hline 6 & $\mathrm{HH}_{2}$ & 0.052 & 0.306 & 0.254 & 4.866 & 64 & 1.04 & 1.01 & 0.97 \\
7 & $\mathrm{HL}_{2}$ & 1.680 & 0.958 & -0.721 & -0.429 & 64 & 0.99 & 1.00 & 1.00 \\
8 & $\mathrm{LH}_{2}$ & 1.680 & 0.958 & -0.721 & -0.429 & 64 & 0.99 & 1.00 & 1.00 \\
9 & $\mathrm{LL}_{2}$ & 53.98 & 56.54 & 2.557 & 0.047 & 64 & 0.93 & 0.93 & 0.99 \\
\hline
\end{tabular}

(b)

\begin{tabular}{|r|l|r|r|r|r|r|r|r|r|}
\hline$k$ & Band & $A_{k, \text { sep }}$ & $A_{k, \text { iso }}$ & $\Delta A_{k}$ & $\frac{\Delta A_{k}}{A_{k, \text { sep }}}$ & $\frac{1}{\alpha_{k}}$ & $G_{k, \text { sep }}$ & $G_{k, \text { iso }}$ & $\tilde{G}_{k}$ \\
\hline 0 & $\mathrm{HH}_{0}$ & 0.002 & 0.030 & 0.027 & 11.01 & 4 & 4.47 & 2.40 & 0.53 \\
1 & $\mathrm{HL}_{0}$ & 0.097 & 0.069 & -0.027 & -0.282 & 4 & 1.78 & 1.94 & 1.08 \\
2 & $\mathrm{LH}_{0}$ & 0.097 & 0.069 & -0.027 & -0.282 & 4 & 1.78 & 1.94 & 1.08 \\
3 & $\mathrm{LL}_{0}$ & 3.802 & 3.830 & 0.027 & 0.007 & 4 & 0.71 & 0.71 & 0.99 \\
\hline 3 & $\mathrm{HH}_{1}$ & 0.020 & 0.100 & 0.079 & 3.862 & 16 & 1.27 & 1.15 & 0.90 \\
4 & $\mathrm{HL}_{1}$ & 0.540 & 0.383 & -0.156 & -0.289 & 16 & 1.03 & 1.06 & 1.02 \\
5 & $\mathrm{LH}_{1}$ & 0.540 & 0.383 & -0.156 & -0.289 & 16 & 1.03 & 1.06 & 1.02 \\
6 & $\mathrm{LL}_{1}$ & 14.10 & 14.45 & 0.343 & 0.024 & 16 & 0.84 & 0.84 & 0.99 \\
\hline 6 & $\mathrm{HH}_{2}$ & 0.237 & 0.622 & 0.384 & 1.618 & 64 & 1.02 & 1.00 & 0.98 \\
7 & $\mathrm{HL}_{2}$ & 3.424 & 2.579 & -0.844 & -0.246 & 64 & 0.98 & 0.98 & 1.00 \\
8 & $\mathrm{LH}_{2}$ & 3.424 & 2.579 & -0.844 & -0.246 & 64 & 0.98 & 0.98 & 1.00 \\
9 & $\mathrm{LL}_{2}$ & 49.34 & 52.02 & 2.679 & 0.054 & 64 & 0.94 & 0.94 & 0.99 \\
\hline
\end{tabular}

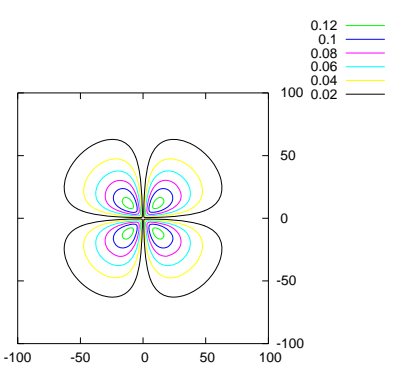

Fig. 2. Contour plot of $\Delta r$ for $\rho=0.95$.

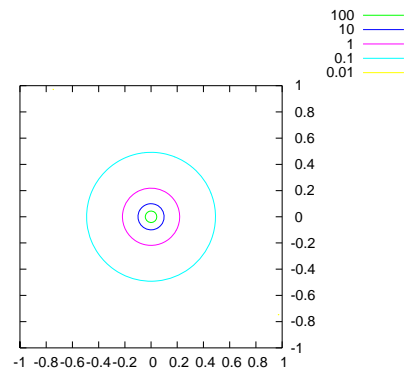

Fig. 4. Contour plot of $\hat{r}_{\text {iso }}$ for $\rho=0.95$.

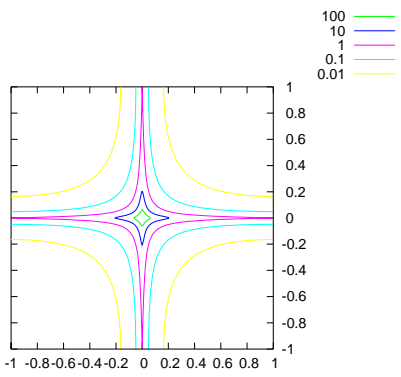

Fig. 3. Contour plot of $\hat{r}_{\text {sep }}$ for $\rho=0.95$.

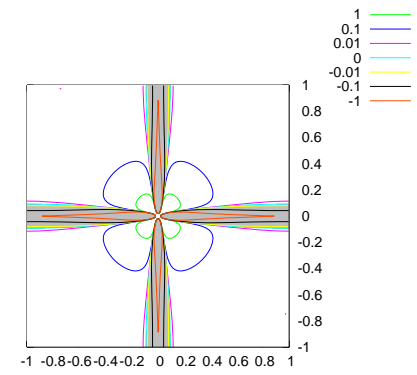

Fig. 5. Contour plot of $\widehat{\Delta r}$ for $\rho=0.95$. 